\title{
Cerebellar Vermis Medulloblastoma
}

National Cancer Institute

\section{Source}

National Cancer Institute. Cerebellar Vermis Medulloblastoma. NCI Thesaurus. Code C5401.

A medulloblastoma arising from the vermis of the cerebellum. 